\title{
ON THE HAMILTONIAN AND LAGRANGIAN FORMULATION OF CLASSICAL DYNAMICS FOR PARTICLES WITH SPIN
}

\author{
TH.W. RUIJGROK and H. VAN DER VLIST \\ Instituut voor Theoretische Fysica, Rijksuniversiteit, Utrecht, The Netherlands*
}

Received 28 November 1979

The classical mechanics of nonrelativistic particles is generalized by also considering the spin components as canonical variables. Poisson-brackets and canonical transformations are discussed. The Lagrangian equations of motion are given and it is shown how rotational invariance leads to well known conservation laws. The method is extended to many dimensional spins and to the classical description of particles with isopin.

\section{Introduction}

In this quantum mechanical century the concept of an intrinsic particle spin is usually considered as foreign to classical mechanics. The same can be said of isotopic spin, as is illustrated in a paper by Wong'), who derives an equation of motion for a "classical" isospin, defined as the quantum mechanical expectation value of the corresponding operator. It is possible, however, to develop a Hamiltonian and a Lagrangian formalism for classical particles with spin, in complete analogy with the canonical theory for spinless particles. Although this possibility has been recognized ${ }^{2}$ ), we consider it worthwhile to show in more detail how the theory can be constructed.

We therefore consider a system of $N$ particles with coordinates $q_{k}$, momenta $\boldsymbol{p}_{k}$ and spins $\boldsymbol{S}_{k}$. The Hamiltonian equations of motion are

$$
\dot{q}_{k}=\left(q_{k}, H\right) ; \quad \dot{p}_{k}=\left(p_{k}, H\right) ; \quad \dot{S}_{k}^{\alpha}=\left(S_{k}^{\alpha}, H\right),
$$

where the time derivative is denoted by a dot. The Poisson-bracket for two functions $A$ and $B$ which are functions of $q_{k}, p_{k}$ and $S_{k}$, is defined as

$$
(A, B)=\sum_{k}\left(\frac{\partial A}{\partial q_{k}} \frac{\partial B}{\partial p_{k}}-\frac{\partial A}{\partial p_{k}} \frac{\partial B}{\partial q_{k}}\right)+\sum_{k} \varepsilon_{\alpha \beta \gamma} \frac{\partial A}{\partial S_{k}^{\alpha}} \frac{\partial B}{\partial S_{k}^{\beta}} S_{k}^{\gamma},
$$

where the summation over Greek indices is implied. The usual properties of

* Mailing address: Princetonplein 5, P.O. Box 80.006, 3508 TA Utrecht, The Netherlands. 
Poisson-brackets, i.e.,

$$
\begin{aligned}
& (A, B)=-(B, A), \\
& \left(A_{1}+A_{2}, B\right)=\left(A_{1}, B\right)+\left(A_{2}, B\right), \\
& (A, B C)=(A, B) C+(A, C) B
\end{aligned}
$$

are easily proved and it is also straightforward, although more elaborate, to prove that the Jacobi-identity

$$
((A, B), C)+((B, C), A)+((C, A), B)=0
$$

is satisfied.

An example of the above equations is given by the Hamiltonian

$$
H=\frac{1}{2 m}\left|p-\frac{e}{c} A\right|^{2}+e \phi-\frac{g e}{2 m c} S \cdot B,
$$

which describes the motion of an atom with charge $e$ and magnetic moment $(g e / 2 m c) S$ in an inhomogeneous electromagnetic field. The equations of motion (1) lead to the well known form

$$
m \ddot{r}=e\left[E+\frac{1}{c} \dot{\boldsymbol{r}} \times \boldsymbol{B}\right]+\frac{g e}{2 m c} \nabla(S \cdot B)
$$

and

$$
\dot{S}=-\frac{g e}{2 m c}(S \times B) .
$$

In the same way equations of motion can be derived for other spinHamiltonians. We mention a few examples

$$
H_{\mathrm{LS}}=\frac{1}{2 m^{2} c^{2}} \frac{1}{r} \frac{\mathrm{d} V}{\mathrm{~d} r}(L \cdot S)
$$

for the description of atomic finestructure

$$
H_{\mathrm{dd}}=\gamma\left\{\frac{\boldsymbol{S}_{1} \cdot \boldsymbol{S}_{2}}{r^{3}}-\frac{3\left(\boldsymbol{r} \cdot \boldsymbol{S}_{1}\right)\left(r \cdot \boldsymbol{S}_{2}\right)}{r^{5}}\right\},
$$

which is important in describing spin-lattice relaxation.

$$
H_{\mathrm{FM}}=-J \sum_{(j, k)_{n n}} S_{j} \cdot S_{\mathrm{k}}
$$

describing a Heisenberg ferromagnet and

$$
H_{\mathrm{MS}}=-J^{1} \sum_{(j, k)_{n n}}\left(S_{j} \cdot S_{k}\right)^{2}
$$

which is the Hamiltonian used by Maier and Saupe in their theory of liquid 
crystals. Many more examples could be given, but it is not the purpose of this paper to study all these spin systems. Instead we will concentrate on the formal aspects and study canonical spin transformations in the next section. In section 3 we give a Lagrangian formulation of the dynamical problem and a discussion of rotational invariance. The paper ends with a generalization to higher dimensional spin and the equations of motion of classical particles with isospin.

\section{Canonical transformations}

In this section we restrict ourselves to a single spin and also the dependence of the Hamiltonian on the coordinates and momenta will be omitted. This is no essential limitation, since all results to be derived in this section can easily be extended to the more general case.

The Poisson-bracket (2) for $A(S)$ and $B(S)$ then becomes

$$
(A, B)=\varepsilon_{\alpha \beta \gamma} \frac{\partial A}{\partial S^{\alpha}} \frac{\partial B}{\partial S^{\beta}} S^{\gamma}
$$

and in particular

$$
\left(S^{\alpha}, S^{\beta}\right)=\varepsilon_{\alpha \beta \gamma} S^{\nu} .
$$

We now define a transformation $S^{\alpha} \rightarrow \tilde{S}^{\alpha}=\tilde{S}^{\alpha}(S)$ to be canonical if for the new spin the relation (4) holds again, i.e.:

$$
\left(\tilde{S}^{\alpha}, \tilde{S}^{\beta}\right)=\varepsilon_{\alpha \beta \gamma} \tilde{S}^{\nu} .
$$

The Poisson-bracket in the left hand side of eq. (5) is to be calculated with differentiations with respect to the old spin components. It can be shown, however, that, like with the $(p, q)$-Poisson-brackets, the spin-brackets are invariant under canonical transformations: Let $A\left(S^{\alpha}\right)=\tilde{A}\left(\tilde{S}^{\alpha}\right)$ and $B\left(S^{\alpha}\right)=$ $\tilde{B}\left(\tilde{S}^{\alpha}\right)$. Then

$$
\begin{aligned}
& (A, B)_{s}=\varepsilon_{\alpha \beta \gamma} \frac{\partial A}{\partial S^{\alpha}} \frac{\partial B}{\partial S^{\beta}} S^{\gamma}=\varepsilon_{\alpha \beta \gamma} \frac{\partial \tilde{S}^{\mu}}{\partial S^{\alpha}} \frac{\partial \tilde{S}^{\nu}}{\partial S^{\beta}} \frac{\partial \tilde{A}}{\partial \tilde{S}^{\mu}} \frac{\partial \tilde{B}}{\partial \tilde{S}^{\nu}} S^{\gamma} \\
& =\left(\tilde{S}^{\mu}, \tilde{S}^{\nu}\right)_{s} \frac{\partial \tilde{A}}{\partial \tilde{S}^{\mu}} \frac{\partial \tilde{B}}{\partial \tilde{S}^{\nu}}=\varepsilon_{\mu \nu \lambda} \frac{\partial \tilde{A}}{\partial \tilde{S}^{\mu}} \frac{\partial \tilde{B}}{\partial \tilde{S}^{\nu}} \tilde{S}^{\lambda}=(\tilde{A}, \tilde{B})_{s} .
\end{aligned}
$$

The indices $S$ and $\tilde{S}$, indicating which are the independent variables, will therefore be omitted from now on.

Also the equation of motion $\dot{S}^{\alpha}=\left(S^{\alpha}, H\right)$ is form invariant under a canonical transformation: 


$$
\begin{aligned}
\dot{\tilde{S}}^{\alpha} & =\frac{\partial \tilde{\boldsymbol{S}}^{\alpha}}{\partial \boldsymbol{S}^{\beta}} \dot{\boldsymbol{S}}^{\beta}=\varepsilon_{\beta \gamma \lambda} \frac{\partial \tilde{S}^{\alpha}}{\partial S^{\beta}} \frac{\partial H}{\partial \boldsymbol{S}^{\gamma}} S^{\lambda}=\varepsilon_{\beta \gamma \lambda} \frac{\partial \tilde{S}^{\alpha}}{\partial S^{\beta}} \frac{\partial \tilde{S}^{\mu}}{\partial S^{\gamma}} S^{\lambda} \frac{\partial \tilde{H}}{\partial \tilde{\boldsymbol{S}}^{\mu}} \\
& =\left(\tilde{S}^{\alpha}, \tilde{\boldsymbol{S}}^{\mu}\right) \frac{\partial \tilde{H}}{\partial \tilde{S}^{\mu}}=\varepsilon_{\alpha \mu \lambda} \frac{\partial \tilde{H}}{\partial \tilde{S}^{\mu}} \tilde{S}^{\lambda}=\left(\tilde{S}^{\alpha}, \tilde{H}\right)_{\tilde{s}}=\left(\tilde{S}^{\alpha}, \tilde{H}\right) .
\end{aligned}
$$

The most general infinitesimal canonical transformation, which preserves the length of the spin, is given by the non-rigid rotation

$$
\tilde{S}^{\alpha}=S^{\alpha}+(\omega \times S)^{\alpha}+O\left(\omega^{2}\right),
$$

in which $\omega=\varepsilon \nabla F$. The generating function $F(S)$ is arbitrary. The proof is rather simple and will not be given here. Instead we will consider the transformation

$$
\tilde{S}^{\alpha}=\lim _{\lambda \rightarrow 1} S^{\alpha}(\lambda)
$$

in which $S^{\alpha}(\lambda)$ is given by

$$
S^{\alpha}(\lambda)=S^{\alpha}+\lambda\left(S^{\alpha}, F\right)+\frac{\lambda^{2}}{2 !}\left(\left(S^{\alpha}, F\right), F\right)+\ldots
$$

We will show that this transformation is canonical and does not change the length of the spin. It is evident that $S^{\alpha}(\lambda)$ as defined in eq. (7) is the unique solution of the differential equation

$$
\frac{\mathrm{d} S^{\alpha}(\lambda)}{\mathrm{d} \lambda}=\left(S^{\alpha}(\lambda), F\right)
$$

under the initial condition $S^{\alpha}(0)=S^{\alpha}$. If we define $G(\lambda)=\left(S^{\alpha}(\lambda), S^{\beta}(\lambda)\right)$, so that $G(0)=\varepsilon_{\alpha \beta \gamma} S^{\gamma}$, then

$$
\begin{aligned}
\frac{\mathrm{d} G}{\mathrm{~d} \lambda} & =\left(\frac{\mathrm{d} S^{\alpha}(\lambda)}{\mathrm{d} \lambda}, S^{\beta}(\lambda)\right)+\left(S^{\alpha}(\lambda), \frac{\mathrm{d} S^{\beta}(\lambda)}{\mathrm{d} \lambda}\right) \\
& =\left(\left(S^{\alpha}(\lambda), F\right), S^{\beta}(\lambda)\right)+\left(S^{\alpha}(\lambda),\left(S^{\beta}(\lambda), F\right)\right) .
\end{aligned}
$$

Using the Jacobi-identity it follows that this is equal to $-\left(\left(S^{\beta}(\lambda), S^{\alpha}(\lambda)\right), F\right)=$ $(G(\lambda), F)$. Therefore $G(\lambda)$ satisfies the differential equation

$$
\frac{\mathrm{d} G}{\mathrm{~d} \lambda}=(G(\lambda), F),
$$

with the initial condition $G(0)=\varepsilon_{\alpha \beta \gamma} S^{\gamma}$. From eq. (8) it follows that the unique solution is given by $G(\lambda)=\varepsilon_{\alpha \beta \gamma} S^{\gamma}(\lambda)$, so that the transformation (7) is indeed canonical.

Consider now the change in the length of the spin:

$$
\frac{\mathrm{d}}{\mathrm{d} \lambda}\left[S^{\alpha}(\lambda) S^{\alpha}(\lambda)\right]=2 S^{\alpha}(\lambda)\left(S^{\alpha}(\lambda), F\right) .
$$


Writing $F(S)=\bar{F}(\boldsymbol{S}(\lambda))$, the Poisson-bracket in the right hand side can also be calculated by considering $S^{\alpha}(\lambda)$ as the independent variables. This gives

$$
\frac{\mathrm{d}}{\mathrm{d} \lambda}\left[S^{\alpha}(\lambda) S^{\alpha}(\lambda)\right]=2 \varepsilon_{\alpha \beta \gamma} S^{\alpha}(\lambda) S^{\gamma}(\lambda) \frac{\partial \bar{F}}{\partial S^{\beta}(\lambda)}=0,
$$

which proves that the length of the spin does not change under the canonical transformation (7). We have therefore shown that $\tilde{S}^{\alpha} \mathrm{d} \tilde{S}^{\alpha}=S^{\alpha} \mathrm{d} S^{\alpha}$, which is analogous to the so called contact transformations, for which $\Sigma_{k} P_{k} \mathrm{~d} Q_{k}=$ $\Sigma_{k} p_{k} \mathrm{~d} q_{k}$.

As in conventional theory the canonical transformations form a group also in the present case. Although we will not give the full proof, we do want to show that the inverse of (7) exists. For that purpose it is sufficient to prove that the Jacobian of the transformation is equal to unity, i.e.

$$
J(\lambda)=\frac{\partial\left(S^{1}(\lambda), S^{2}(\lambda), S^{3}(\lambda)\right)}{\partial\left(S^{1}, S^{2}, S^{3}\right)}=1 .
$$

Taking the derivative of the determinant for $\lambda=0$ and using eq. (8), it is easy to show that

$$
\left(\frac{\mathrm{d} J}{\mathrm{~d} \lambda}\right)_{\lambda=0}=\frac{\partial\left(S^{\alpha}, F\right)}{\partial S^{\alpha}}=\varepsilon_{\alpha \beta \gamma} \frac{\partial}{\partial S^{\alpha}}\left(\frac{\partial F}{\partial S^{\beta}} S^{\gamma}\right)=0 .
$$

Eq. (9) now follows immediately, because $J(0)=1$.

From the group property it follows that two successive canonical transformations with generators $F$ and $G$ can be combined into one canonical transformation with another generator $H$. Upon substitution and use of the Jacobi-identity it can then be shown that the first few terms of the BakerCampbell-Hausdorff formula are given by

$$
H=F+G+\frac{1}{2}(F, G)+\ldots
$$

Since it is not our purpose to rewrite Whittaker's treatise for spin systems, we will not pursue the subject of canonical transformations any further.

\section{The Lagrange formalism}

In this section we want to show that the equations of motion (1) for a pure many spin system can be derived from the action principle $\delta A=0$, where $A=\int_{t_{1}^{2}}^{t_{2}} L \mathrm{~d} t$ and where the Lagrangian is given by

$$
\begin{aligned}
& L\left(S_{1}, \ldots, S_{N}, \dot{S}_{1}, \ldots, \dot{S}_{N}\right)=\sum_{k} M^{\alpha}\left(S_{k}\right) \dot{S}_{k}^{\alpha}-H\left(S_{1}, \ldots, S_{N}\right) \\
+ & \frac{1}{2} \sum_{k} \lambda_{k}\left(S_{k}^{\alpha} S_{k}^{\alpha}-S_{0}^{2}\right) .
\end{aligned}
$$


The function $\mathbf{M}(\mathbf{S})$ is still to be determined. The term with the multipliers $\lambda_{k}$ is added in order to ensure that each spin has the prescribed length $S_{0}$.

Variation with respect to the functions $\lambda_{k}(t)$ and $S_{k}^{\alpha}(t)$ leads to the EulerLagrange equations $\left|S_{k}(t)\right|=S_{0}$ and $(\mathrm{d} / \mathrm{d} t)\left(\partial L / \partial \dot{S}_{k}^{\alpha}\right)-\partial L / \partial S_{k}^{\alpha}=0$. The latter becomes

$$
\dot{S}_{k} \times \boldsymbol{K}\left(\boldsymbol{S}_{k}\right)+\nabla_{k} H-\lambda_{k} \boldsymbol{S}_{k}=0,
$$

where we have introduced the vector $\mathbf{K}(\mathbf{S})=-\nabla \times \mathbf{M}(\mathbf{S})$. On external multiplication by $\boldsymbol{S}_{k}$ eq. (11) gives

$$
\left(\boldsymbol{K}\left(\boldsymbol{S}_{k}\right) \cdot \boldsymbol{S}_{k}\right) \dot{\boldsymbol{S}}_{k}-\left(\boldsymbol{S}_{k} \cdot \dot{\boldsymbol{S}}_{k}\right) \boldsymbol{K}\left(\boldsymbol{S}_{k}\right)+\boldsymbol{S}_{k} \times \nabla_{k} H=0 .
$$

The middle term is zero because the length of the spin is constant in time. By choosing $\mathbf{M}(\mathbf{S})$ in such a way that

$$
K(S) \cdot S=1,
$$

eq. (12) becomes

$$
\dot{S}_{k}=-S_{k} \times \nabla_{k} H,
$$

which is the same as the Hamilton eq. (1). Taking the scalar product of eq. (11) with $S_{k}$ gives

$$
\lambda_{k}=\boldsymbol{K}\left(\boldsymbol{S}_{k}\right) \cdot \nabla_{k} H \text {. }
$$

Since $\lambda_{k} S_{k}$ is the reaction force which maintains the length of $S_{k}$, the right hand side of eq. (15) is duly proportional to the gradient of $H$ with respect to this $\boldsymbol{S}_{k}$. Substitution of eqs. (14) and (15) into eq. (11) gives an identity, so that (14) and (15) are fully equivalent to (11). The remaining problem is to find $\mathbf{M}(\mathbf{S})$ so that eq. (13) is satisfied. It is certainly not determined uniquely, because $\mathbf{M}^{\prime}\left(\mathbf{S}_{k}\right)=\mathbf{M}\left(\mathbf{S}_{k}\right)+\nabla_{k} \chi\left(\mathbf{S}_{k}\right)$ gives a Lagrangian which differs from the original one by the total derivative

$$
\sum_{k} \frac{\mathrm{d} \chi\left(S_{k}\right)}{\mathrm{d} t}
$$

and therefore gives the same equations of motion. The great arbitrariness in $M(S)$ can also be seen from the following construction.

Consider a sphere with radius $S$, not necessarily equal to $S_{0}$ and cover it with closed contours $C_{k}$ which do not intersect each other. See fig. 1. The contours can be given a direction by making a puncture in the sphere at one point $P$. In each point $\mathbf{S}$ of the sphere we now define $\mathbf{M}(\mathbf{S})$ as the vector tangent to the sphere and to the curve $C_{k}$ through that point, with the direction opposite to that of the curve and a magnitude $|M|=\left(S \Omega_{k} / L_{k}\right)$, where $L_{k}$ is the length of the curve and $\Omega_{k}$ the space angle spanned by $C_{k}$. M 


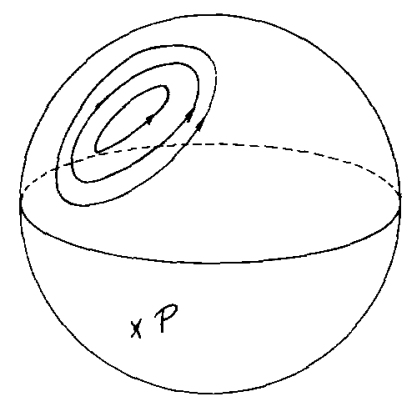

Fig. 1. Construction of $\boldsymbol{M}(\boldsymbol{S})$.

is singular in the point $P$ where $L_{k}=0$ and $\Omega_{k}=4 \pi$. On other spheres the curves $C_{k}^{\prime}$ and the point $P^{\prime}$ are obtained by a continuous change. In this way $\mathbf{M}(\mathbf{S})$ is completely defined in the three dimensional spin space, except for a half line from the origin to infinity, where $\mathbf{M}$ is singular. With Stokes' theorem we now have for any of the curves $C_{k}$

$$
S \Omega_{k}=-\oint_{C_{k}} \boldsymbol{M} \cdot \mathrm{d} \boldsymbol{S}=-S \int_{\Omega_{k}}(\nabla \times \boldsymbol{M}) \cdot \boldsymbol{S} \mathrm{d} \Omega=S \int_{\Omega_{k}}(\boldsymbol{K} \cdot \boldsymbol{S}) \mathrm{d} \Omega .
$$

Since the equality $\int_{\Omega}(K \cdot S) \mathrm{d} \Omega=\Omega$ holds, not only for $C_{k}$ and $\Omega_{k}$, but for any closed curve, we can conclude that the vector $\boldsymbol{K}(\boldsymbol{S})$ constructed from this $\boldsymbol{M}(\boldsymbol{S})$ satisfies eq. (13). A specially simple case is obtained by taking for $C_{k}$ parallel circles perpendicular to a given vector $\boldsymbol{S}_{0}$. Then

$$
\boldsymbol{M}(\boldsymbol{S})=\frac{\boldsymbol{S} \times \boldsymbol{S}_{0}}{\boldsymbol{S} \boldsymbol{S}_{0}+\boldsymbol{S} \cdot \boldsymbol{S}_{0}} .
$$

The corresponding vector $K(S)$ is

$$
K(S)=-\nabla \times M=\frac{1}{S} \frac{S_{0} S+S S_{0}}{S S_{0}+S \cdot S_{0}}
$$

and it is easily checked that $K \cdot S=1$. The form of (16) obtained by taking $S_{0}$ in the positive $z$-direction was arrived at by Jevicki and Papanicolaou ${ }^{3}$ ), in a paper in which a Lagrangian formulation for a classical spin was given. They observe that the Lagrangian is not manifestly invariant under rotations. The same can be said of our expression for the term $\boldsymbol{M} \cdot \boldsymbol{S}$, because $\boldsymbol{M}$ does not transform as a vector. In eq. (16) $S_{0}$ stays fixed and in the more general case the curves $C_{k}$ are fixed (like an external magnetic field) and do not change with a rotation of the spins.

In order to understand this better we consider the behaviour of a part of the Lagrangian $L_{M}=\Sigma_{k} M\left(S_{k}\right) \cdot \dot{S}_{k}$ under an infinitesimal rigid rotation $S_{k}^{\prime \alpha}=$ 
$S_{k}^{\alpha}+\varepsilon_{\alpha \beta \gamma} \omega_{\beta} S \gamma+\mathcal{O}\left(\omega^{2}\right)$. A straight-forward calculation, in which $\boldsymbol{K} \cdot \boldsymbol{S}=1$ is used, shows that $L_{M}^{\prime}=L_{M}+\mathrm{d} \Omega / \mathrm{d} t+\mathcal{O}\left(\omega^{2}\right)$, in which

$$
\Omega=-\boldsymbol{\omega} \cdot \sum_{k}\left\{\boldsymbol{M}\left(\boldsymbol{S}_{k}\right) \times \boldsymbol{S}_{k}+\boldsymbol{S}_{k}\right\}
$$

The action and therefore the equations of motion are thus rotational invariant, provided of course $H\left(S_{1}, \ldots, S_{N}\right)$ is invariant. Using Noether's theorem the function $\Omega$ can be used to construct a conserved quantity ${ }^{4}$ )

$$
\psi=\sum_{k}\left(\frac{\partial L}{\partial \dot{S}_{k}^{\alpha}} \delta S_{k}^{\alpha}\right)-\Omega
$$

We find $\psi=\omega \cdot \Sigma_{k} S_{k}$ and since $\omega$ is arbitrary the total spin vector is conserved. This result can of course also be proved directly from the Hamilton equations of motion. We believe, however, that it is illuminating to see that the breaking of the manifest rotational invariance of the Lagrangian is necessary but harmless.

The Lagrangian formulation given in this section can easily be extended to include the orbital motion of a system of particles.

\section{Generalizations}

It would be very desirable to give a Lagrangian formulation for the relativistic motion of a classical particle with spin in an external electromagnetic field. For a homogenous field the equations of motion have been given by Bargmann, Michel and Telegdi ${ }^{5}$ ). Efforts to generalize their equations to the case of inhomogeneous fields have, however, always led to a strange classical Zitterbewegung, to self-accelerating solutions or to other difficulties $^{6}$ ).

We will not try to improve upon this situation. It is possible, however, to derive very general relativistically invariant equations of motion for particles, not with spin, but with other internal degrees of freedom, like isospin for example.

With space-time and momentum variables $x^{\alpha}$ and $p^{\alpha}(\alpha=0,1,2,3)$, with isospin variables $I^{a}(a=1,2,3)$ and with a metric $-g^{00}=g^{11}=g^{22}=g^{33}=1$, we can define Poisson-brackets which satisfy the usual conditions as follows:

$$
(A, B)=g^{\alpha \beta}\left(\frac{\partial A}{\partial x^{\alpha}} \frac{\partial B}{\partial p^{\beta}}-\frac{\partial A}{\partial p^{\alpha}} \frac{\partial B}{\partial x^{\beta}}\right)+\varepsilon_{a b c} \frac{\partial A}{\partial I^{a}} \frac{\partial B}{\partial I^{b}} I^{c} .
$$

Given a "Hamiltonian" $\mathscr{H}\left(x^{\alpha}, p^{\alpha}, I^{a}\right)$, the equations of motion can be taken as $\dot{F}=\mathrm{d} F / \mathrm{d} \tau=(F, \mathscr{H})$, in which $\tau$ is the eigen time. As an example we will take a 
particle with isospin moving in a given $\mathrm{SU}(2)$ gauge field $f_{\mu \nu}^{a}=$ $\partial_{\mu} b_{\nu}^{a}-\partial_{\nu} b_{\mu}^{a}+g \varepsilon_{a b c} b_{\mu}^{b} b_{\nu}^{c}$, which is derived from potentials $b_{\mu}^{a}$ and satisfy the field equations ${ }^{7}$ )

$$
\partial_{\mu} f_{a}^{\mu \nu}+g \varepsilon_{a b c} b_{\mu}^{b} f^{\mu \nu c}=-j_{v}^{a} .
$$

By taking the Hamiltonian as $\mathscr{H}=(1 / 2 m) \Pi^{\alpha} \Pi_{\alpha}$, where $I I^{\alpha}=p^{\alpha}-g b_{a}^{\alpha} I^{a}$, the canonical equations of motion become

$$
\begin{aligned}
& m \ddot{x}^{\mu}=g\left(f^{\mu \nu} \cdot \boldsymbol{I}\right) \dot{x}_{v}, \\
& \dot{\boldsymbol{I}}=g\left(\boldsymbol{I} \times \boldsymbol{b}^{\mu}\right) \dot{x}_{\mu} .
\end{aligned}
$$

These are the equations first suggested by Wong') on the basis of a quantum mechanical averaging.

In our opinion the advantage of the present derivation is that it is placed in a consistent classical framework.

The same equations are obtained as the Euler-Lagrange equations of a variational principle with as Lagrangian

$$
L=\boldsymbol{M}(\boldsymbol{I}) \cdot \dot{\boldsymbol{I}}+\frac{1}{2} m \dot{x}^{\alpha} \dot{\boldsymbol{x}}_{\alpha}+g \dot{x}^{\alpha} I^{a} b_{\alpha}^{a}+\frac{1}{2} \lambda\left(\boldsymbol{I} \cdot \boldsymbol{I}-I_{0}^{2}\right),
$$

where $M$ is defined as in the previous section. For $\lambda$ we find

$$
\lambda=-g\left(\boldsymbol{K} \cdot \boldsymbol{b}_{\alpha}\right) \dot{x}^{\boldsymbol{a}} .
$$

As a last example of the canonical formalism we want to consider $n$ dimensional spins described by $S_{\alpha \beta}=-S_{\beta \alpha}(\alpha, \beta=1,2, \ldots, n)$. Since these spins are the generators of rotations in the $\alpha-\beta$ plane of a Euclidean space the Poisson-brackets should be defined by

$$
\left(S_{\mu \nu}, S_{\alpha \beta}\right)=\delta_{\mu \alpha} S_{\nu \beta}+\delta_{\nu \beta} S_{\mu \alpha}-\delta_{\nu \alpha} S_{\mu \beta}-\delta_{\mu \beta} S_{\nu \alpha},
$$

which can be extended to brackets for two arbitrary fuctions of $S_{\alpha \beta}$. As an example we consider a linear chain of these $n$-dimensional spins having a Heisenberg type of interaction described by

$$
H=\frac{1}{2} J \sum_{k} S_{\alpha \beta}^{k} S_{\beta \alpha}^{k+1}+\frac{1}{2} H_{\alpha \beta} \sum_{k} S_{\beta \alpha}^{k},
$$

where the last term describes the influence of a magnetic field $H_{\alpha \beta}$. In this case the equations of motion

$$
\frac{\mathrm{d} S_{\mu \nu}^{k}}{\mathrm{~d} t}=\left(S_{\mu \nu}^{k}, H\right)
$$

take the following form:

$$
\frac{\mathrm{d} S_{\mu \nu}^{k}}{\mathrm{~d} t}=J\left(S_{\mu \alpha}^{k-1}+S_{\mu \alpha}^{k+1}\right) S_{\alpha \nu}^{k}-J S_{\mu \alpha}^{k}\left(S_{\alpha \nu}^{k-1}+S_{\alpha \nu}^{k+1}\right)+H_{\mu \alpha} S_{\alpha \nu}^{k}-S_{\mu \alpha}^{k} H_{\alpha \nu}
$$


The length of each spin tensor $\left\|S^{k}\right\|^{2}=-S_{\alpha \beta}^{k} S_{\beta \alpha}^{k}$ is constant in time and the total magnetization $M_{\mu \nu}=\Sigma_{k} S_{\mu \nu}^{k}$ satisfies the equation $\mathrm{d} M_{\mu \nu} / \mathrm{d} t=$ $H_{\mu \alpha} M_{\alpha \nu}-M_{\mu \alpha} H_{\alpha \nu}$.

The physical content of these equations will not be investigated any further, since in this paper we are only concerned with exhibiting a new way of treating classical spin systems. The same holds for our closing remark that a Lagrangian formulation can also be given for a classical spin density $\boldsymbol{S}(\boldsymbol{r}, t)$. The path integral formalism can then be used for the quantization of such a field ${ }^{8}$ ).

\section{References}

1) S.K. Wong, Nuovo Cimento 65A (1970) 689.

2) See e.g., H.A. Kramers, Quantum Mechanics (North-Holland, Amsterdam, 1957) p. 236. N.D. Mermin, Phys. Rev. 134A (1964) 112 Appendix A.

E.C.G. Sudarshan and N. Mukunda, Classical Dynamics: A Modern Perspective (John Wiley, New York, 1974) p. 318.

3) A. Jevicki and N. Papanicolaou, Annals of Physics (N.Y.) 120 (1979) 107.

4) For a simple derivation see: N.G. van Kampen, Reports on Mathematical Physics 3 (1972) 235.

5) V. Bargmann, L. Michel and V.L. Telegdi, Phys. Rev. Lett. 2 (1959) 435.

6) Of the vast literature on the subject we only quote F. Halbwachs, Progr. Theor. Physics 24 (1960) 291 and A.J. Hanson and T. Regge, Annals of Physics (N.Y.) 87 (1974) 498.

7) C.N. Yang and R.L. Mills, Phys. Rev. 96 (1954) 191.

8) See e.g. ref. 3. 